This article was downloaded by: [Jansson-Fröjmark, Markus]

On: 11 December 2008

Access details: Access Details: [subscription number 906619265]

Publisher Routledge

Informa Ltd Registered in England and Wales Registered Number: 1072954 Registered office: Mortimer House, 37-41 Mortimer Street, London W1T 3JH, UK

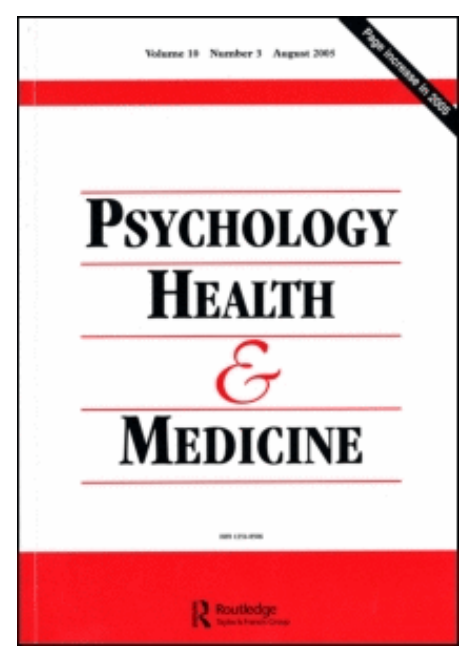

Psychology, Health \& Medicine

Publication details, including instructions for authors and subscription information:

http://www.informaworld.com/smpp/title content=t713441652

\title{
Exploratory factor analysis of the Modified Somatic Perception Questionnaire on a sample with insomnia symptoms
}

Markus Jansson-Fröjmark a; Shane MacDonald a

aepartment of Behavioral, Social, and Legal Sciences, Örebro University, Örebro, Sweden

Online Publication Date: 01 January 2009

To cite this Article Jansson-Fröjmark, Markus and MacDonald, Shane(2009)'Exploratory factor analysis of the Modified Somatic Perception Questionnaire on a sample with insomnia symptoms',Psychology, Health \& Medicine, 14:1,62 — 72

To link to this Article: DOI: $10.1080 / 13548500802001793$

URL: http://dx.doi.org/10.1080/13548500802001793

\section{PLEASE SCROLL DOWN FOR ARTICLE}

Full terms and conditions of use: http://www.informaworld.com/terms-and-conditions-of-access.pdf

This article may be used for research, teaching and private study purposes. Any substantial or systematic reproduction, re-distribution, re-selling, loan or sub-licensing, systematic supply or distribution in any form to anyone is expressly forbidden.

The publisher does not give any warranty express or implied or make any representation that the contents will be complete or accurate or up to date. The accuracy of any instructions, formulae and drug doses should be independently verified with primary sources. The publisher shall not be liable for any loss, actions, claims, proceedings, demand or costs or damages whatsoever or howsoever caused arising directly or indirectly in connection with or arising out of the use of this material. 


\title{
Exploratory factor analysis of the Modified Somatic Perception Questionnaire on a sample with insomnia symptoms
}

\author{
Markus Jansson-Fröjmark* and Shane MacDonald \\ Department of Behavioral, Social, and Legal Sciences, Örebro University, SE-701 82 Örebro, Sweden
}

(Received 19 April 2007; accepted 20 February 2008)

Objectives: The purpose of this study was to examine the factorial solution of the Modified Somatic Perception Questionnaire (MSPQ) among individuals with insomnia symptoms in the general population.

Design: A cross-sectional study with a randomly selected sample from the general population $(N=3600 ; 20-60$ year old $)$ was used. In total, 251 of the 2179 respondents fulfilled the criteria for insomnia symptoms and filled out a survey on demographic parameters, the MSPQ, the Hospital Anxiety and Depression Scale, sleep medication use and health care consumption.

Methods: Exploratory factor analysis and correlations were used.

Results: The results showed that a two-factor solution, accounting for $47.31 \%$ of the variance, was extracted from the 13 items of the MSPQ. Although one factor consisting of 10 items determined general symptoms of somatic arousal $(\alpha=0.83)$, the other factor with three items assessed stomach symptoms and nausea $(\alpha=0.78)$. The two factors were significantly inter-correlated $(r=0.54)$ and significantly associated with the total MSPQ $(r=0.96, r=0.74)$. The two factors also showed discriminant validity with anxiety and depression and predictive validity with retrospective reports of sleep medication use and health care consumption. A few significant interactions emerged the two MSPQ factors and degree of sleep complaints.

Conclusions: Although it is often assumed that the MSPQ taps a single factor of somatic arousal, this study on individuals with insomnia symptoms suggests that a two-factor solution has the best fit. Further research on the factorial solution of the MSPQ is warranted.

Keywords: Modified Somatic Perception Questionnaire; MSPQ; exploratory factor analysis; insomnia

\section{Introduction}

In 1983, the Modified Somatic Perception Questionnaire (MSPQ) was presented as a tool for the assessment of chronic pain (Main, 1983). The MSPQ consists of 13 items and it is often assumed that the MSPQ determines one factor tapping symptoms of somatic arousal. Since the MSPQ was introduced in the literature in 1983, the instrument has been used extensively in clinical and research settings. The MSPQ has mainly been employed on individuals suffering from various pain conditions (Adams, Mannion, \& Dolan, 1999; Donceel \& Du Bois, 1999; Gockel et al., 1995; Greenough, 1993; Koho, Aho, Watson, \& Hurri, 2001; Main, 1983; Norrefalk, Svensson, Ekholm, \& Borg, 2005; Penta \& Fraser,

*Corresponding author. Email: markus.jansson@bsr.oru.se 
1997; Trief, Grant, \& Fredrickson, 2000; Wand et al., 2004). The instrument has however also been used on tinnitus patients (Newman, Wharton, \& Jacobson, 1997; Robinson et al., 2003), on patients with Meniere's disease (Storper, Spitzer, \& Scanlan, 1998), on bereaved couples (Lang, Gottlieb, \& Amsel, 1996), on individuals with insomnia (Jansson \& Linton, 2007), on women with chest pain (Warner, 1995) and on patients with myocardial infarction (Frasure-Smith \& Lespérance, 2003). The MSPQ has thus over the years become an oft-used measure in clinical and research settings.

Although the MSPQ has been widely used in clinical and research settings, there has yet been no thorough attempt to investigate the factorial solution of the instrument. When the MSPQ has been employed in investigations, the instrument has consistently been used as if it assesses a single construct (i.e. the items are summed). This notion of that the MSPQ determines a single factor is problematic since no study has yet examined the factorial solution of the MSPQ. Although the original article briefly reported on the internal consistency and factorial loadings of the MSPQ (Main, 1983), there are several problems with how the factorial solution was explored in that study. For example, there is no information available in the original article about whether preparatory analysis was performed to ensure that the characteristics of the data set were suitable for the factor analysis to be conducted. Further, information is also lacking about which factor extraction method that was used, how the number of factors that was retained was decided upon, and which rotation method that was employed. This is problematic since several authors recommend certain guidelines to be followed when factor analysis is to be performed (Costello \& Osborne, 2005; Fabrigar, Wegener, MacCallum, \& Strahan, 1999; Tabachnick \& Fidell, 2001). For example, in one article discussing the recommendations for factor analysis, the authors advise the use of maximum likelihood as the factor extraction method, oblique rotation and screen plots plus multiple test runs for information on how many factors might be in a particular data set (Costello \& Osborne, 2005). An improved understanding of the factorial solution of the MSPQ would thus benefit from the use of current guidelines on how to perform a factor analysis.

The purpose of the present study was therefore to investigate the factorial solution of the MSPQ among individuals with insomnia symptoms in the general population. The factor analysis was performed using recent guidelines (e.g. Costello \& Osborne, 2005). Thus, the following features were employed: maximum likelihood as the factor extraction method, oblique rotation, screen plots and multiple test runs (Costello \& Osborne, 2005). The sample size was chosen to optimise the likelihood of identifying underlying factors (Costello \& Osborne, 2005). Since the investigation of the psychometric properties of the MSPQ is still in its infancy, exploratory factor analysis was used (Tabachnick \& Fidell, 2001). An advantage of exploratory factor analysis is that it can consolidate variables and generate hypotheses about underlying processes, which can then be tested in future studies using both exploratory and confirmatory factor analysis.

\section{Aim of this study}

The overall purpose of this investigation was to examine the factorial solution of the MSPQ among individuals with insomnia symptoms in the general population. The aim was also to explore the internal consistency of the retained factor(s), the discriminant validity of the retained factor(s) with anxiety and depression, the predictive validity of the retained factor(s) to sleep medication use and sleep-related health care consumption, and the association between the retained factor(s) and degree of sleep complaints. 


\section{Method}

\section{Overview of the design}

This study was carried out in the general population. A random sample of 3600 residents in middle Sweden was administered questions assessing demographic factors, sleep, the MSPQ, the Hospital Anxiety and Depression Scale (HADS), sleep medication use and health care consumption. The Örebro Hospital's Board on Research Ethics approved this study.

\section{Participants}

The study is a population-based investigation from a random sample of 3600 residents, 20 60 years old, from four counties in middle Sweden. The age range was chosen as to reflect individuals in the workforce. The sample was selected from the population registers of the four counties. Of the 3600 residents, 2179 participants (61\%) returned the questionnaire. Of the 2179 participants that returned the questionnaire, $251(11.5 \%)$ reported insomnia symptoms. The 251 individuals with insomnia symptoms were the focus of this study and the demographic parameters for the study participants are presented in Table 1.

\section{Procedure}

The questionnaire was based on validated instruments and was mailed to the random sample of 3600 residents, along with a letter of introduction, information about the project and a stamped return-envelope. If a response was not received within 2 weeks a reminder was mailed. If an additional 2 weeks elapsed without a response a second reminder was sent.

\section{Measures}

The following domains were assessed via the questionnaire: demographic factors, sleep medication use, health care consumption, sleep, the MSPQ and the HADS. The following

Table 1. Overview of the 251 study participants with insomnia symptoms.

\begin{tabular}{lc}
\hline & The study participants \\
\hline Age (years; mean) & $44(11)$ \\
Gender (female) & $64 \%$ \\
Civil status (married or cohabitant) & $73 \%$ \\
Occupational status & \\
Employment & $68 \%$ \\
Unemployment & $9 \%$ \\
Student & $9 \%$ \\
On sick leave or pension & $14 \%$ \\
Education (highest) & \\
Compulsory school & $23 \%$ \\
High school & $37 \%$ \\
College or university & $22 \%$ \\
Other education & $18 \%$ \\
Sleep medication use the past 12 months & $28 \%$ \\
Health care consumption the past 12 months & $22 \%$ \\
\hline
\end{tabular}

Means are presented with standard deviations in parenthesis. 
demographic parameters were determined: age, gender, civil status (married or cohabitant living alone), level of education (compulsory school - high school - college or university other education) and occupational status (employed - students - unemployed - sick leave or pension). Sleep medication use was assessed by a single item: "Have you used any prescribed sleep medication during the past 12 months?" (yes/no). Health care consumption was determined by a single question: "Have you sought health care (e.g. a physician or a nurse) due to disturbed sleep during the past 12 months?" (yes/no).

Items concerning sleep were taken from the Basic Nordic Sleep Questionnaire (Partinen \& Gislason, 1995) and the Uppsala Sleep Inventory (Liljenberg, Almqvist, Hetta, Roos \& Ågren, 1988). The perception of a sleep difficulty during the past 3 months (“Have you had problems sleeping during the past three months?') was assessed, and if a "yes" response was reported, the participant was asked to assess how many nights per week this had occurred during the past 3 months. Further, these two questions were used to assess sleep initiation difficulties and sleep maintenance difficulties: "On average: How many minutes are you awake before you fall asleep?" and "On average: If you wake up at night, how many minutes are you awake?" The measures are considered to be psychometrically sound and suitable to be employed in epidemiological research on insomnia (Liljenberg et al., 1988; Linton \& Bryngelsson, 2000). In this study, insomnia symptoms were defined as reporting a sleep problem during the past 3 months for three nights or more per week and sleep initiation or maintenance difficulties (i.e. 30 min or more on at least one of the difficulties).

The MSPQ (Main, 1983) was administered to measure somatic arousal. The MSPQ is a self-rating scale in which thirteen somatic symptoms are rated on 4-point scales $(1=$ not all; $4=$ very much). The total score ranges from 13 to 52 . The participant is asked to rate how often he or she has experienced somatic symptoms during the past week. Investigations have shown that the MSPQ is a psychometrically sound instrument with adequate validity and reliability (Deyo, Walsh, Schoenfeld, \& Ramamurthy, 1989; Main, 1983). The HADS (Zigmond \& Snaith, 1983) was used to assess anxiety and depression. The HADS is a self-rating instrument in which the severity of anxiety and depression is rated on 4-point scales (0-3). Seven questions are related to depression and seven to anxiety, both with a score range of $0-21$. Investigations have shown that the HADS is a psychometrically sound instrument, e.g. good internal consistency and a two-factor solution (Herrmann, 1997).

\section{Statistical analysis}

To reveal any latent variables within the MSPQ that cause the manifest variables to covary, exploratory factor analysis was used in line with recommendations. An exploratory factor analysis was used since the study of the psychometric properties of the MSPQ is still in its infancy. To ensure that the characteristics of the data set were suitable for the factor analysis to be conducted on the insomnia sample $(N=251)$, the Kaiser-Meyer-Olkin (KMO) measure of sampling adequacy and the Bartlett Test of Sphericity (BTS) were conducted on the data. A maximum likelihood factor extraction procedure with oblique rotation (direct oblimin) was employed since this approach is particularly useful in extracting psychologically meaningful factors and because of the possibility that the extracted factors may be correlated. The screen test and multiple test runs were used to decide the number of meaningful factors that might be in the data set. The minimum loading of an item was determined at 0.32 . To investigate internal reliability, Cronbach's alpha was used, and 0.70 was considered as the minimum acceptable criterion of 
instrument internal reliability (Kline, 1993). The discriminant validity was examined with Pearson's Correlation Coefficient (MSPQ and its retained factor(s) with anxiety and depression) and the predictive validity was investigated with Eta correlation statistic and logistic regression (MSPQ and its retained factor(s) with sleep medication use and health care consumption). Analysis of variance was used to examine whether there were differences between the retained factor(s) and aspects of sleep complaints and their distinct groups.

\section{Results}

\section{Exploratory factor analysis}

For the 251 study participants with insomnia symptoms, the number of valid responses on the MSPQ items ranged from 241 and 249. All items were checked for normality and were found to be sufficiently normally distributed for further analyses. To examine the interdependence among the 13 items, Pearson's Correlation Coefficient was used. These analyses showed that the correlations among the items ranged from 0.13 to 0.64 . In size, $49 \%$ of the correlations were small $(0.10-0.30), 44 \%$ moderate $(0.30-0.50)$ and $7 \%$ large (above 0.50 ). The KMO measure of sampling adequacy yielded an index of 0.85 and the BTS was significant $\left(\chi_{(\mathrm{df}=78)}^{2}=1054.68, p<0.001\right)$. This preparatory analysis confirmed that the data distribution satisfied the psychometric criteria for the factor analysis to be performed.

To assess the underlying structure for the 13 items of the MSPQ, factor extraction with maximum likelihood and oblimin rotation was employed. To check for stability of parameter estimates, the sample was first divided into two random halves $\left(n_{1}=123\right.$, $n_{2}=128$ ). In both the sub-samples and in the sample as a whole, the screen test implied a two-factor solution. Given the established stability of estimates, the sample as a whole was used in further analyses. Table 2 displays the 13 MSPQ items and item loadings for the two-factor solution for the sample. In the table, the means and standard deviations for the

Table 2. Exploratory factor analysis of the Modified Somatic Perception Questionnaire $(N=251)$.

\begin{tabular}{lrrrr}
\hline & $M$ & $S D$ & $\begin{array}{r}\text { Factor 1: Item } \\
\text { loadings }\end{array}$ & $\begin{array}{r}\text { Factor 2: Item } \\
\text { loadings }\end{array}$ \\
\hline Mouth becoming dry (1-4) & 1.96 & 1.11 & 0.435 & -0.069 \\
Blurring of vision (1-4) & 1.74 & 0.99 & 0.544 & 0.001 \\
Sweating all over (1-4) & 2.06 & 1.11 & 0.633 & -0.050 \\
Stomach churning (1-4) & 2.44 & 1.13 & 0.097 & 0.582 \\
Muscles twitching and jumping (1-4) & 1.73 & 1.03 & 0.446 & 0.063 \\
Feeling hot all over (1-4) & 2.02 & 1.11 & 0.650 & -0.029 \\
Feeling faint (1-4) & 2.55 & 1.06 & 0.610 & 0.025 \\
Muscles in neck aching (1-4) & 2.57 & 1.24 & 0.469 & 0.019 \\
Dizziness (1-4) & 1.98 & 1.10 & 0.726 & -0.010 \\
Tense feeling across forehead (1-4) & 2.15 & 1.16 & 0.523 & 0.046 \\
Legs feeling weak (1-4) & 1.86 & 1.10 & 0.703 & 0.015 \\
Nausea (1-4) & 1.75 & 1.03 & 0.220 & 0.542 \\
Pain or ache in stomach (1-4) & 2.16 & 1.11 & -0.058 & 1.027 \\
Factor 1: 3 items (1-12) & 6.35 & 2.66 & & \\
Factor 2: 10 items (10-40) & 20.51 & 6.93 & & \\
MSPQ: 13 items (13-52) & 26.86 & 8.67 & & \\
\hline
\end{tabular}

Factor $1: 37.71 \%$ of the variance. Factor $2: 9.60 \%$ of the variance. 
13 MSPQ items, the two factors and the total MSPQ are also shown in the table. The factor analysis demonstrating a two-factor solution showed a variance accounted for of $47.31 \%$ (Eigen value: 6.15 ). The first factor with 10 items, determining general symptoms of somatic arousal, accounted for $37.71 \%$ of the variance. The second factor consisting of 3 items, assessing stomach symptoms and nausea, accounted for $9.60 \%$ of the variance.

Additional factor analyses were run to investigate the validity of the two-factor solution. To test the potential appropriateness of a one-factor and a three-factor solution in the sample, additional factor analyses were run and the number of factors to retain was set manually at one factor and three factors, respectively. The results of these analyses showed that the one-factor solution accounted for accumulatively $37.71 \%$ of the variance $(\alpha=0.85)$. The three-factor solution accounted for accumulatively $56.52 \%$ of the variance $\left(\alpha_{1}=0.80\right.$, $\alpha_{2}=0.74$ and $\alpha_{3}=0.77$ ), but it contained one low-loading item ("Mouth becoming dry") and one factor with only two items. To further examine the appropriateness of the two-factor solution, additional factor analyses were run. In the two analyses, exploratory factor analysis was employed on men $(n=132)$ and women $(n=236)$, respectively. The screen test implied a two-factor solution for both men and women. For men, the two-factor solution accounted for $46.74 \%$ and for women $48.12 \%$ of the variance. In all, these analyses suggested that a two-factor solution had the best fit for the data.

\section{Internal consistency, discriminant validity and predictive validity of the two factors}

Factor inter-correlations and correlations with the total MSPQ, coefficient alphas and item-total correlations were computed for the two factors. As can be seen in Table 3, the two factors were significantly and moderately inter-correlated $(r=0.54)$, suggesting that the two factors measure distinct, but related, constructs. Both the factors were significantly and highly associated with the total MSPQ (first factor: $r=0.96$; second factor: $r=0.74$ ). The coefficient alpha for the first factor was 0.83 and for the second factor 0.78 . The alpha values for the two factors thus exceeded the criterion for acceptable instrument internal reliability of 0.70 (Kline, 1993). The item-total correlations for the ten items with the first factor were between 0.55 and 0.72 and for the three items with the second factor between 0.76 and 0.89 .

The discriminant validity of the retained two factors with anxiety and depression (HADS) was examined. As is displayed in Table 3, the results from correlational analyses showed that the correlations between the first factor and anxiety $(r=0.41, p<0.001)$

Table 3. Correlations between the two retained MSPQ factors, the total MSPQ, anxiety, depression, sleep medication use, and health care consumption: 251 participants with insomnia symptoms.

$\begin{array}{llllll}1 & 2 & 3 & 4 & 5 & 6\end{array}$

1. Factor $1(\mathrm{MSPQ})^{\mathrm{a}}$

2. Factor $2(\mathrm{MSPQ})^{\mathrm{b}}$

3. MSPQ: Total

4. Anxiety (HADS)

5. Depression (HADS)

6. Sleep medication use

7. Health care consumption

$0.54^{* *}$
$0.96^{* *}$
$0.41^{* *}$
$0.36^{* *}$
$0.14^{* *}$
$0.16^{* *}$

$0.74 * *$

$0.39 * *$

$0.37 * *$

$0.15^{* *}$

$0.45^{* *}$
$0.40^{* *}$
$0.16^{* *}$
$0.14^{* *}$

$0.63 * *$

0.08

$0.12^{*}$

$0.12 * \quad 0.09$

$0.59 * *$

Pearson's correlation coefficient, Eta, and Phi statistics were used.

${ }^{\text {a }}$ Factor with 10 items determining general symptoms of somatic arousal.

${ }^{\mathrm{b}}$ Factor with three items assessing stomach symptoms and nausea. ${ }^{*} p<0.05,{ }^{* *} p<0.01$. 
and depression $(r=0.36, p<0.001)$ were significant at a moderate level. The correlations between the second factor and anxiety $(r=0.39, p<0.001)$ and depression $(r=0.37$, $p<0.001)$ were also significant at a moderate level.

The predictive validity of the two retained factors was investigated with sleep medication use and sleep-related health care consumption as the outcome variables. First, the two factors were correlated (Eta) with prescribed sleep medication use during the past 12 months (yes/no). As can be seen in Table 3, the results showed that the first factor $(\eta=0.15, p<0.01)$ and the second factor $(\eta=0.14, p<0.01)$ were significantly correlated with prescribed sleep medication use. In all, a high score on the two factors were significantly correlated with a reported use of prescribed sleep medication during the past 12 months. Second, the two factors were correlated (Eta) with sleep-related health care consumption during the past 12 months (yes/no). Although the first factor was significantly correlated with health care consumption $(\eta=0.16, p<0.01)$, the second factor was not significantly correlated with health care consumption $(\eta=0.08)$. In all, a high score on the first factor was significantly correlated with a reported consumption of sleep-related health care during the past 12 months. To examine whether the two factors were significantly associated with sleep medication use and health care consumption in a multivariate model, the two retained factors were added in combination with age, gender, anxiety and depression using logistic regression. The results showed that only age was significantly related to sleep medication use $(p<0.001)$ and health care consumption $(p=0.028)$; the older the individuals were, the more likely they were to report using sleep medication and consuming health care.

\section{Association between degree of sleep complaints and level of somatic arousal}

To assess the relationship between the two MSPQ factors and degree of sleep complaints, three aspects of sleep complaints were categorised accordingly: sleep onset latency into three distinct groups (less than $30 \mathrm{~min}, 30-60 \mathrm{~min}$ and more than $60 \mathrm{~min}$ ), wake time after sleep onset into three distinct groups (less than $30 \mathrm{~min}, 30-60 \mathrm{~min}$ and more than $60 \mathrm{~min}$ ), and frequency into two distinct groups (3-5 days per week and 7 days per week). The mean level of somatic arousal (the two retained MSPQ factors) was then calculated for the three aspects of sleep complaints and their distinct groups. These findings are presented in Table 4. Analysis of variance was used to examine whether there were differences between the three aspects of sleep complaints and their distinct groups on level of somatic arousal. The results showed that there was a significant interaction between sleep onset latency and the second MSPQ factor $(F=3.18, p=0.044)$; the two groups reporting a higher degree of sleep onset problems had a significantly higher level of somatic arousal on the second factor compared with the group with the lowest degree of sleep onset problems. Also, a significant interaction emerged between frequency and the first factor $(F=9.75$, $p<0.001)$; the group reporting more frequent sleep problems had a higher level of somatic arousal on the first factor than the group with less frequent sleep problems. There were no significant differences between the sleep onset groups on the first MSPQ factor $(p=0.64)$, the sleep maintenance groups on either the first factor $(p=0.75)$ or the second factor $(p=0.92)$, and the frequency groups on the second factor $(p=0.77)$.

\section{Discussion}

This investigation examined the factorial solution of the MSPQ among individuals with insomnia symptoms in the general population. The exploratory factor analysis 
Table 4. The association between degree of sleep complaints and level of somatic arousal.

\begin{tabular}{lcc}
\hline & MSPQ: Factor 1 & MSPQ: Factor 2 \\
\hline Sleep onset latency & & \\
$\quad<30 \min (n=54)$ & 19.8 & 5.9 \\
$30-60 \min (n=115)$ & 20.9 & 6.2 \\
$\quad>60 \min (n=82)$ & 20.6 & 7.0 \\
Wake after sleep onset & & 6.5 \\
$\quad<30 \min (n=64)$ & 20.9 & 6.3 \\
$30-60 \min (n=99)$ & 20.6 & 6.3 \\
$\quad>60$ min $(n=88)$ & 20.1 & 6.3 \\
Frequency & 19.1 & 6.4 \\
3-5 days/week $(n=121)$ & 21.9 & \\
7 days/week $(n=130)$ & & \\
\hline
\end{tabular}

Means are presented in the table.

demonstrated that a two-factor solution had the best fit for the data, accounting for approximately $47 \%$ of the variance. Although one factor that appeared determined general symptoms of somatic arousal $(37.71 \%$ of the variance; 10 items: $\alpha=0.83)$, the other factor assessed stomach symptoms and nausea $(9.60 \%$ of the variance; 3 items: $\alpha=0.78$ ). The results from the correlational analyses suggested that the two factors were significantly inter-correlated and significantly associated with the total MSPQ. Analyses of discriminant validity showed that the two factors were significantly and moderately related to anxiety and depression. Analyses of predictive validity demonstrated that the two factors were significantly associated with sleep medication use and health care consumption (except for the second factor and health care consumption). The results also pointed towards some significant interactions between the two MSPQ factors and degree of sleep complaints. The overall picture of this study suggests therefore tentatively that the MSPQ captures two underlying constructs among individuals with insomnia symptoms.

The finding of a two-factor solution for the MSPQ among individuals with insomnia symptoms might be interpreted in several ways. One possibility is simply that a two-factor solution has the best fit for the data, at least for individuals with insomnia symptoms. Interestingly, the two factors were conceptually distinct; while one factor assessed general symptoms of somatic arousal, the other factor determined stomach symptoms and nausea. If the notion of a two-factor solution is correct, the use of a one-factor approach to the MSPQ should then perhaps be discouraged. It is however important to keep in mind that the internal consistency for the one-factor solution was high, which supports the view that the MSPQ might be viewed as a single construct measure. Given that the present study was the first to thoroughly investigate the factorial solution of the MSPQ and documented a two-factor solution, the possibility of a two-factor approach to the MSPQ cannot be discarded. If the two-factor solution has any bearing, this also means that the results of this study are not in line with the findings in the original article (Main, 1983), which resulted in a one-factor solution. Given that the original article did not document whether preparatory analysis was used, which factor extraction method that was employed, how the number of factors that was retained was decided upon, and which rotation method that was used, it is difficult to compare the two attempts to examine the factorial solution of the MSPQ. 
A second possibility to how the two-factor solution can be interpreted is that the underlying constructs for the MSPQ differs between conditions. In that case, the MSPQ possibly captures a single factor among patients with pain problems and two distinct constructs among individuals with insomnia symptoms. Since this study and the original article (Main, 1983) are the only investigations of the factorial solution of the MSPQ, it is difficult to speculate about what the MSPQ captures among individuals with other conditions, e.g. tinnitus and myocardial infarction. However, given that the present study and the original article documented two differing factorial solutions for individuals with insomnia symptoms and pain, the notion of separate factorial solutions between conditions cannot be discarded.

Future research is clearly needed to further explore the factorial solution of the MSPQ. It is important to underscore that factor analysis is an error-prone procedure even with large samples and optimal data (Costello \& Osborne, 2005). The present results and the findings from the original article (Main, 1983) should therefore be interpreted with caution. With that in mind, it is also warranted to emphasise that the sample in the current study (insomnia symptoms in a general population sample) may be different from the original sample (chronic back pain) on important characteristics, which, in turn, may explain the different factor solution. Future research is therefore warranted to shed light on the factorial solution of the MSPQ for individuals with insomnia, pain and other conditions, using both exploratory and confirmatory factor analysis. It is also suggested to employ the larger set of MSPQ items from the original study for future investigations. Such research might be carried out in the general population and in clinical settings on individuals with different conditions.

The present investigation also examined the two retained MSPQ factors' internal consistency, discriminant and predictive validity, and association with sleep complaints. The results indicated that the two factors were significantly inter-correlated and significantly related to the total MSPQ. Noteworthy, the first factor, assessing general symptoms of somatic arousal, had a very high correlation with the 13-items MSPQ and was also more strongly associated with the total MSPQ than the second factor. The two factors were also significantly associated with anxiety and depression, indicating that the factors are related but also distinct to anxiety and depression. Tentatively, these results might be interpreted in that the MSPQ is distinct from what the HADS measures, i.e. behavioural, cognitive and emotional aspects of anxiety and depression. Analyses of predictive validity demonstrated that the two factors were significantly related to prescribed sleep medication use during the past 12 months, and that the first factor was significantly associated with health care consumption during the past 12 months. These findings might cautiously be viewed as evidence for the two factors ability to predict criterion measures for individuals with insomnia symptoms. Before firm conclusions are however drawn on the factors' predictive validity, this needs to be tested longitudinally. Finally, the results pointed towards some significant interactions between the two MSPQ factors and degree of sleep complaints (i.e. sleep onset latency and the second MSPQ factor and frequency of sleep complaints and the first factor). This might tentatively be viewed as evidence for a relationship between more pronounced sleep complaints and a higher score on the MSPQ.

An important question pertains to how the MSPQ should be employed in future clinical and research settings. When the MSPQ has been employed in previous studies, the instrument has consistently been used as if it assesses a single construct. The present study implies that the MSPQ might possibly be viewed upon as an instrument that captures two underlying constructs. However, given that there have only two attempts to explore the 
factorial solution of the MSPQ, the shift from a one-factor to a two-factor approach appears too hasty. Instead, the MSPQ should be put under scientific scrutiny to further revise and evaluate the instruments' factorial solution.

This study must be interpreted in light of certain limitations. One limitation of this study was the participation rate at $61 \%$. Although this is not uncommon in this type of research, it may restrict the generalisability of the findings. A second limitation was that retrospective reports were used when assessing sleep medication use and health care consumption. A stronger test of the relationship between the two MSPQ factors and important criterion measures for individuals with insomnia would be to use a longitudinal design. A third limitation was that the existence of insomnia symptoms was determined solely based on self-report measures. This limits the generalisability to objective instruments. A related limitation was also that the self-report measures did not fully capture the concept of insomnia in a clinical sense (e.g. American Psychiatric Association, 1994). It is therefore possible to generalise the findings to insomnia on a symptomatic level.

Taken as a whole, this investigation on individuals with insomnia symptoms showed that the MSPQ appears to capture two underlying constructs. Although one factor determined general symptoms of somatic arousal, the other factor assessed stomach symptoms and nausea. Given the error-proneness of factor analysis, the lack of data on the factorial solution of the MSPQ, and the current use of MSPQ as a single factor instrument, more research is clearly warranted before firm conclusions can be drawn.

\section{Acknowledgement}

We express our appreciation to the Swedish Council for Working Life and Social Research for financial support and to the Department of Psychiatry at the Orebro University Hospital for supporting the preparation of this manuscript.

\section{References}

American Psychiatric Association (1994). Diagnostic and statistical manual of mental disorders (4th ed.). Washington, DC: American Psychiatric Association.

Adams, M.A., Mannion, A.F., \& Dolan, P. (1999). Personal risk factors for first-time low back pain. Spine, 24, 2497-2505.

Costello, A.B., \& Osborne, J.W. (2005). Best practices in exploratory factor analysis: four recommendations for getting the most from your analysis. Practical Assessment Research \& Evaluation, 10, 1-9.

Deyo, R.A., Walsh, N.E., Schoenfeld, L.S., \& Ramamurthy, S. (1989). Studies of the Modified Somatic Perceptions Questionnaire (MSPQ) in patients with back pain: psychometric and predictive properties. Spine, 14, 507-510.

Donceel, P., \& Du Bois, M. (1999). Predictors for work incapacity continuing after disc surgery. Scandinavian Journal of Work, Environment \& Health, 25, 264-271.

Fabrigar, L.R., Wegener, D.T., MacCallum, R.C., \& Strahan, E.J. (1999). Evaluating the use of exploratory factor analysis in psychological research. Psychological Methods, 4, 272-299.

Frasure-Smith, N., \& Lesperance, F. (2003). Depression and other psychological risks following myocardial infarction. Archives of General Psychiatry, 60, 627-636.

Gockel, M., Lindholm, H., Alaranta, H., Viljanen, A., Lindquist, A., \& Lindholm, T. (1995). Cardiovascular functional disorder and stress among patients having neck-shoulder symptoms. Annals of the Rheumatic Diseases, 54, 494-497.

Greenough, C.G. (1993). Recovery from low back pain 1-5 year follow-up of 287 injury-related cases. Acta Orthopaedica Scandinavica, 254, 1-34.

Herrmann, C. (1997). International experiences with the Hospital anxiety and depression scale - a review of validation data and clinical results. Journal of Psychosomatic Research, 42, 17-41.

Jansson, M., \& Linton, S.J. (2007). Psychological mechanisms in the maintenance of insomnia: arousal, distress, and sleep-related beliefs. Behaviour Research and Therapy, 45, 511-521. 
Kline, P. (1993). The handbook of psychological testing. Routledge: London.

Koho, P., Aho, S., Watson, P., \& Hurri, H. (2001). Assessment of chronic pain behaviour: reliability of the method and its relationship with perceived disability, physical impairment and function. Journal of Rehabilitation Medicine, 33, 128-132.

Lang, A., Gottlieb, L.N., \& Amsel, R. (1996). Predictors of husbands' and wives' grief reactions following infant death: the role of marital intimacy. Death Studies, 20, 33-57.

Liljenberg, B., Almqvist, M., Hetta, J., Roos, B.E., \& Agren, H. (1988). The prevalence of insomnia: the importance of operationally defined criteria. Annals of Clinical Research, 20, 393-398.

Linton, S.J., \& Bryngelsson, I.L. (2000). Insomnia and its relationship to work and health in a working-age population. Journal of Occupational Rehabilitation, 10, 169-183.

Main, C.J. (1983). The Modified Somatic Perception Questionnaire (MSPQ). Journal of Psychosomatic Research, 27, 503-514.

Newman, C.W., Wharton, J.A., \& Jacobson, G.P. (1997). Self-focused and somatic attention in patients with tinnitus. Journal of the American Academy of Audiology, 8, 143-149.

Norrefalk, J.R., Svensson, O., Ekholm, J., \& Borg, K. (2005). Can the back-to-work rate of patients with long-term non-malignant pain be predicted? International Journal of Rehabilitation Research, 28, 9-16.

Partinen, M., \& Gislason, T. (1995). Basic Nordic Sleep Questionnaire (BNSQ): a quantitated measure of health and dysfunction. Journal of Sleep Research, 4, 150-155.

Penta, M., \& Fraser, R.D. (1997). Anterior lumbar interbody fusion. A minimum 10-year follow-up. Spine, 22, 2429-2434.

Robinson, S.K., McQuaid, J.R., Viirre, E.S., Betzig, L.L., Miller, D.L., Bailey, K.A., et al. (2003). Relationship of tinnitus questionnaires to depressive symptoms, quality of well-being, and internal focus. International Tinnitus Journal, 9, 97-103.

Storper, I.S., Spitzer, J.B., \& Scanlan, M. (1998). Use of glycopyrrolate in the treatment of Meniere's disease. Laryngoscope, 108, 1442-1445.

Tabachnick, B.G., \& Fidell, L.S. (2001). Using multivariate statistics. Boston: Allyn and Bacon.

Trief, P.M., Grant, W., \& Fredrickson, B. (2000). A prospective study of psychological predictors of lumbar surgery outcome. Spine, 25, 2616-2621.

Wand, B.M., Bird, C., McAuley, J.H., Dore, C.J., MacDowell, M., \& De Souza, L.H. (2004). Early intervention for the management of acute low back pain: a single-blind randomized controlled trial of biopsychosocial education, manual therapy, and exercise. Spine, 29, 2350-2356.

Warner, C.D. (1995). Somatic awareness and coronary artery disease in women with chest pain. Heart \& Lung, 24, 436-443.

Zigmond, A.S., \& Snaith, R.P. (1983). The Hospital anxiety and depression scale. Acta Psychiatrica Scandinavica, 67, 361-370. 